\title{
Nonlinear System Identification by Pareto RBF Network Ensemble Based on Evolutionary Multiobjective Optimization
}

\author{
Nobuhiko Kondo, Toshiharu Hatanaka and Katsuji Uosaki \\ Department of Information and Physical Science, Osaka University \\ Yamadaoka 2-1, Suita, 562-0871, Japan \\ E-mail: \{nobuhiko,hatanaka\}@ist.osaka-u.ac.jp
}

\begin{abstract}
Radial basis function (RBF) network's structure determination method by using multiobjective evolutionary algorithms is considered and an ensemble network constructed by the Pareto optimal networks is also proposed in this paper. The candidates of RBF network structure are encoded into the binary coded chromosomes. Then, they evolve toward Pareto optimal front which is defined by the several objective functions corresponding to the model accuracy and model complexity. The application to the nonlinear system identification is studied by numerical simulations, the applicability of the proposed approach is discussed.
\end{abstract}

\section{Introduction}

In the control system design, fault detection, fault diagnosis, signal processing, time series prediction and other engineering problems, the mathematical models of an actual system have important roles. System modeling of unknown objective systems based on the observed input and output data is a fundamental issue of engineering problem and it is called as system identification [1]. System identification techniques have been developed for the linear systems, however, almost actual existing systems may have inherent nonlinear properties such as dead zone and saturation, so linear system models are not enough to represent such dynamics of nonlinear systems. Then, nonlinear system identification has been studied and a lot of identification algorithms have been developed in the recent years [2].

Almost approaches of the system identification give one best model under some criterion based on the given prior knowledge or information. However, the system model optimized under the specific criterion is not always the optimal model because there are usually several demands to a system model. For example, it required that the model should be easy to handle and well explainable for the modeling data set contaminated by observation noise, but these properties are mutually exclusive. Hence, system identification method based on multiobjective optimization will be an useful tool, but there are a few studies from this viewpoint $[3,4]$. The multiobjective optimization is receiving much at- tentions in the field of system optimization and multiobjective evolutionary algorithms are much being studied as efficient technique to providing the Pareto optimum solutions with a single run $[5,6]$. Nonlinear system identification by using multiobjective evolutionary algorithms have been proposed, these approaches deal with polynomial dynamic system model and give the optimal model set concerning model accuracy and complexity $[7,8]$.

On the other hand, artificial neural networks have wide variety of applications due to their powerful nonlinear mapping ability, the use of artificial neural networks is being studied as promising approach to nonlinear system identification $[9,10]$. Though the primary importance in applying neural network to nonlinear system modeling is to select its structure suitably, a general method of the structure determination has not established, because the optimum structure depends on a class of the objective system and learning algorithm. So the network structure is generally determined by trial and error or a heuristic method.

From this viewpoint, we have been considering multiobjective optimization based nonlinear system modeling using the evolutionary algorithms. In this paper, we deal with the static nonlinear system modeling using RBF (Radial Basis Function) network, which is a kind of artificial neural network. RBF network has in their hidden layer a number of basis function which respond locally in input space. The network output is the linear sum of the basis function values. If the parameters of RBF networks, i.e. the number of basis functions and the widths and centers of each basis function, are determined, output layer weights can be calculated with the training data [11]. This parameter setting affects the quality of function approximation. Therefore we consider the structure determination problem of RBF networks as a multiobjective optimization problem that concerns with the model accuracy, the model complexity and the output layers' weights. Then a method of obtaining the candidates of model as a Pareto optimal set based on evolutionary algorithms is proposed. The designers will be able to select one model from the Pareto optimal set obtained by the proposed method according to their use or a specific criteria. By introduc- 
ing the concept of ensemble learning $[12,13]$ and neural network ensembles based on Pareto set [14, 15, 16], one system model can be obtained by constructing the neural network ensemble network.

In this paper, we consider the nonlinear system identification as multiobjective optimization problem and identification technique by using the Pareto optimum RBF networks ensemble is proposed. Numerical simulation studies are carried out to show the applicability of the proposed technique to nonlinear system identification without using the specific prior knowledge or information about the actual systems.

\section{Multiobjective Genetic Algorithms and Pareto RBF Networks}

\subsection{Genetic Algorithm}

GA (Genetic algorithm) is a kind of stochastic search or optimization method, originally proposed by Holland [17] which has been invented based on natural genetics and evolution. The outline of simple GA procedure is following way. Initially, the initial population of individuals having a binary digit string as the "chromosome" is generated randomly. Each bit of chromosome is called "gene". The "fitness", which is a measure of adaptation to environment, is calculated for each individual. Then, "selection" operation leaving individuals to next generation is performed based on fitness value, and then "crossover" and "mutation" are performed on the selected individuals to generate new population by transforming chromosomes into offspring's ones. This procedure is continued until the end condition is satisfied. This algorithm is conforming to the mechanism of evolution, in which the genetic information changes for every generation and the individuals which adapt to environment better survive preferentially. Since GA is a stochastic parallel search and GA requires only fitness value based on the objective functions, GA attracts attentions as a solver of multiobjective optimization problems due to parallel search.

\subsection{Multiobjective Optimization}

In the actual problems, there generally exists tradeoff among the objective functions. And so two concept, "domination" and the "Pareto optimum", are considered in multiobjective optimization.

Let's consider the multiobjective optimization problem such as

$$
\begin{array}{rc}
\max & f_{1}(\boldsymbol{x}), \cdots, f_{n}(\boldsymbol{x}) \\
\text { subject to } & g_{j}(\boldsymbol{x}) \geq 0, j=1,2, \cdots, k
\end{array}
$$

where, $x$ represents $m$ dimensional decision variable $x=\left(x_{1}, x_{2}, \cdots, x_{m}\right)^{T}$ and $f_{i}(x), \quad i=1,2, \cdots, n$ denote $n$ objective functions. $g_{i}(x), \quad i=1,2, \cdots, k$ are the constraint conditions.

First, $x_{1}$ is said to "dominate" $x_{2}$, if and only if

$$
\forall i=1,2, \ldots, n \quad f_{i}\left(x_{1}\right) \leq f_{i}\left(x_{2}\right)
$$

and

$$
\exists j=1,2, \ldots, n \quad f_{j}\left(x_{1}\right)<f_{j}\left(x_{2}\right) .
$$

Then, $x_{0}$ which is not dominated by any other $x$ is called the "Pareto optimal solution". Pareto optimal solution is considered to be the best solution comprehensively. And generally many Pareto optimal solutions exist simultaneously. Considering tradeoff among the objective functions, on multi-objective optimization problems it is important to obtain a Pareto optimal solution set.

\subsection{Multi-objective GA based on rank}

A parameter rank is introduced in order to apply the concepts of domination and Pareto optimum to GA. Though there are some ranking methods, this study adopts Fonseca's ranking method [5]. According to Fonseca's ranking method, a rank of an individual $x_{i}$ on a generation $t$ is:

$$
\operatorname{rank}\left(\boldsymbol{x}_{i}, t\right)=1+p_{i}^{(t)}
$$

where $p_{i}$ is the total number of individuals which dominate $x_{i}$. By calculating this rank for each individ-

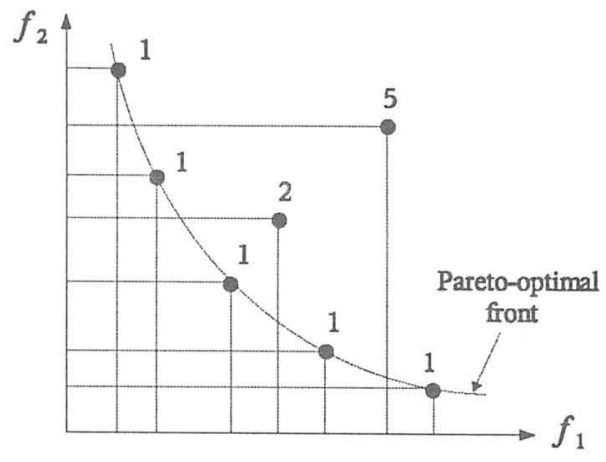

Fig. 1: Fonseca's ranking method

ual and selecting based on it, a population can evolve toward a Pareto optimal solution set. Since GA is a multi-point search algorithm, GA is expected to find a Pareto optimal set in a single simulation run.

\subsection{RBF Network}

$\mathrm{RBF}$ (Radial Basis Function) network is constructed of three layers as shown in Fig.2 and has basis functions which respond locally in input space. Basis function $\phi_{j}(x)$ in this study is defined by Gaussian function,

$$
\phi_{j}(x)=\exp \left(-\frac{\left(x-c_{j}\right)^{T}\left(x-c_{j}\right)}{2 \sigma_{j}^{2}}\right)
$$

Here, $x$ is input variable, $\boldsymbol{c}_{j}$ is center vector, and $\sigma_{j}^{2}$ is a parameter which decides function width. Using this $\phi_{j}(x)$, RBF network is constructed as:

$$
u(\boldsymbol{x})=w_{0}+\sum_{j=1}^{m} w_{j} \phi_{j}(\boldsymbol{x})
$$


Here, $m$ is the number of hidden units, i.e., the basis functions, and $w_{j}$ are the output layer weights. RBF network will be determined if the parameters $m, c_{j}, \sigma_{j}$, and $w_{j}$ are estimated based on the data observed from the system. In this study, these parameters are estimated by two GAs. The parameters $\sigma_{j}$ are assumed to be constant value for simplicity.

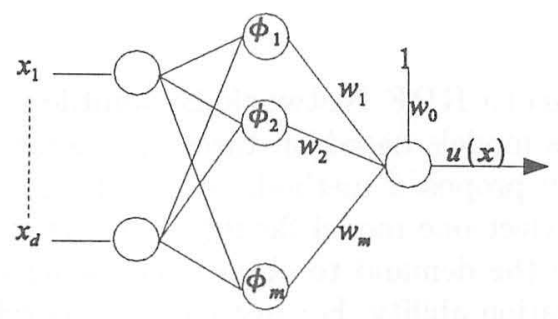

Fig. 2: RBF network

\subsection{Genetic Representation}

In this study, we apply MOGA (Multi-Objective Genetic Algorithm), based on NSGA-II proposed by Deb [6] to determine the both of the number of basis functions and the centers of them. The candidate of the center of basis function is assumed to be the position of the training data points. The chromosomes of MOGA population indicate that the data points are employed as centers of basis functions i.e. "1" represents that a basis function is located at the corresponding training data point, as shown in Fig.3. By this setting, the length of the chromosome becomes equal to the number of training data, the number of " 1 " gene in the chromosome indicates the number of basis functions and the locus of the " 1 " shows the center position of the basis functions. Then, the connection weights are estimated

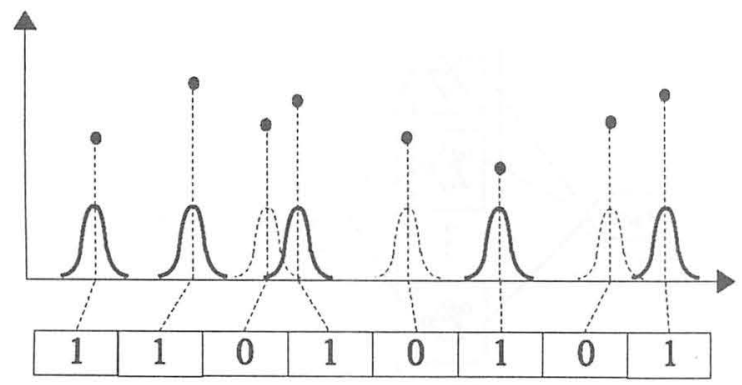

Fig. 3: Chromosome representation

by real-coded GA, each individual represents directly the weight values. The first stage is multi-objective binary coded GA to examine the structure candidate of the $\mathrm{RBF}$ network and the second stage is real coded GA that estimates the connection weights for the candidate of RBF network structure represented by each individual in the first stage.

The overall flow diagram is shown in Fig.4, in which two-stage GAs are used to RBF network determination.

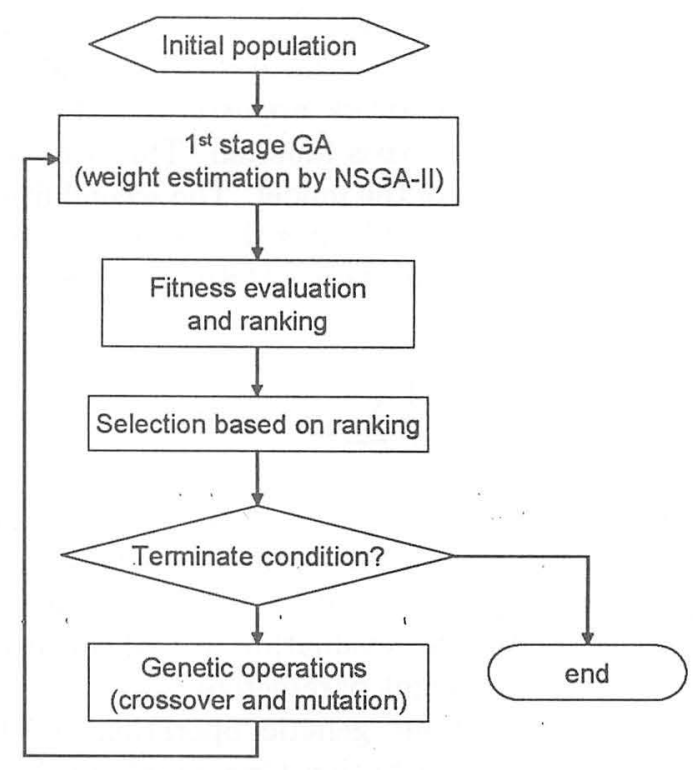

Fig. 4: Overall flowchart of the proposed approach

\subsection{Construction of Pareto RBF Networks}

The Pareto optimal RBF network construction algorithm is consist of multi-objective genetic algorithm including real-coded GA. As multi-objective genetic algorithm we adopt NSGA-II which is one of multi-objective genetic algorithms and is known to have the capability to maintain diversity.

After estimating all the parameters of the network, rank is assigned for each individual by the concept of multi-objective optimization problem, in which three fitness functions are to be minimized. Then Pareto optimal individuals will be obtained in accordance with NSGA-II algorithm. In NSGA-II, we apply genetic operations which are the uniform crossover and the bit reversal mutation.

\section{Problem Statement}

Let's consider following single output single input nonlinear system

$$
\begin{aligned}
y_{t} & =g\left(\phi_{t}\right)+e_{t} \\
\phi_{t} & =\left(y_{t-1}, \ldots, y_{t-n_{y}}, u_{t-1}, \ldots, u_{t-n_{u}}\right)^{T}
\end{aligned}
$$

where, $g(\cdot)$ represents unknown nonlinear function, $y_{t} \in$ $\Re$ is system output, $u_{t} \in \Re$ is system input and $e_{t} \in \Re$ is noise term with zero mean and infinite variance. $\phi_{t}$ is called as a regression vector composed of delayed input and output, $n_{y}$ is unknown maximum delay of output and $n_{u}$ is unknown maximum delay of input. 


\subsection{Evaluation}

It is generally demanded that the mathematical models not only can explain the relationship between input and output enough but also is simple in order to have the generalization ability. Then in this study three evaluation criteria are set for the evaluation of MOGA which determines the network architecture. The first fitness is the number of basis function. This fitness indicates the complexity of the model. The second fitness is $\log M S E$. This fitness indicates the extent of a fit of the model to the training data. MSE(Mean Squared Error) is defined as :

$$
M S E=\frac{1}{n} \sum_{i=1}^{n}\left\{y_{i}-\hat{y}_{i}\right\}^{2}
$$

Here, $y_{i}$ is the observed output, $\hat{y}_{i}$ is the model output. The third fitness is the sum of the absolute value of weights. These three evaluation criteria are to be minimized. $M S E$ is used for evaluation in real-coded GA which estimates the weight parameters.

In real-coded GA, about genetic operation, UNDX (Unimodal Normal Distribution Crossover) [18] is applied in the proposed method. UNDX generates two offsprings by normal random numbers which is determined by three parents, as shown in Figure 5. Basically offsprings are generated by normal distribution around segment connecting two parents. The third parent is used to determine the standard deviation of normal distribution.

MGG[19] is adopted as the generation alternation

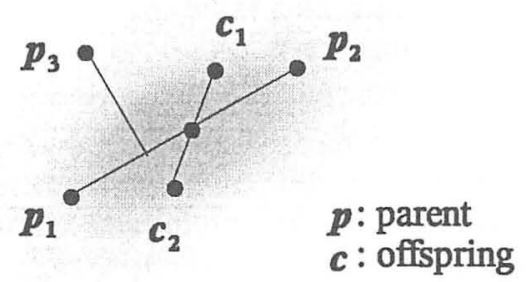

Fig. 5: UNDX

model of real-coded GA used in the proposed method. MGG is said to have an ability to preserve the diversity of population. MGG procedure is as follows.

1. Plurality of real number vector is generated at random as the initial population.

2. Two parents are selected at random from population.

3. $2 n_{c}$ offsprings are generated by applying UNDX to two parents $n_{c}$ times. Here the third parent which determines the standard deviation of normal distribution is selected from population.

4. Fitness values of each offspring are calculated, then two individuals are selected from the set which is composed of two parents and all offsprings, then two parents are replaced by the selected two individual. The individuals selected here are elite and the individual selected by roulette selection in which the elite was pruned.

5. Continue $2 \sim 4$ until the end condition is met.

The procedure of the proposed method is shown in Fig.4.

\subsection{Pareto RBF Network Ensemble}

Various models based on three criterion can be obtained by proposed method, so the designers will be able to select one model flexibly. On the other hand, there are the demand to obtain one model with good generalization ability. For instance, model selection by information criteria has been studied.

Recently the ensemble learning is receiving much attentions in the field of machine learning. In the ensemble learning, a monolithic model is constructed by combining several models. While some learning methods to make models constructing ensemble have been proposed, in this study ensemble is constructed of Pareto optimal models obtained by the proposed method.

Suppose that the number of Pareto models is $m$ and the output of $j$-th network is $y_{j}(x)$, then the output of ensemble network $y^{E N}(x)$ is :

$$
y^{E N}(\boldsymbol{x})=\sum_{j=1}^{m} w_{j} y_{j}(\boldsymbol{x})
$$

Here, $w_{j}$ is the weight on the output of $j$-th network. In this study, $w_{j}$ is assumed to be $1 / m$ about every $j$, for simplicity.

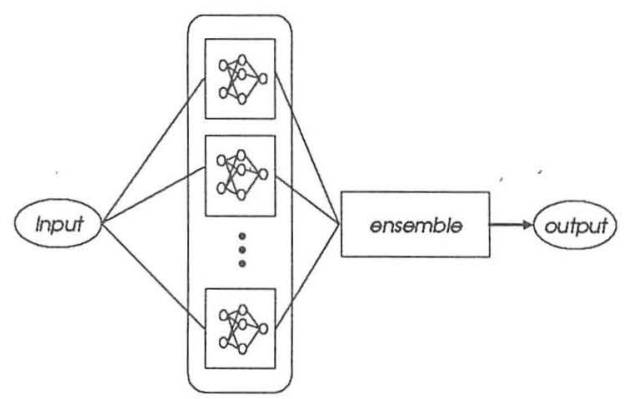

Fig. 6: RBF network ensemble

\section{Numerical examples}

To show the effect of the proposed approach we consider following examples.

\subsection{Simulation 1}

We assume that the true system is described by

$$
y_{t}=f\left(y_{t-1}, y_{t-2}, y_{t-3}, u_{t-1}, u_{t-2}\right)+e_{t} .
$$


Where,

$$
f\left(x_{1}, x_{2}, x_{3}, x_{4}, x_{5}\right)=\frac{x_{1} x_{2} x_{3} x_{5}\left(x_{3}-1\right)+x_{4}}{1+x_{2}^{2}+x_{3}^{2}},
$$

and $e_{y}$ indicates observation noise with mean 0 and variance $\sigma_{\varepsilon}^{2}$. Then, $u_{t}, t=1,2, \cdots$ are sampled by uniform distribution $U[-1,1]$ we obtained 50 set of input and output observation data. The control parameters for genetic algorithms are listed in the Table1, we carried out simulation runs in the case of $\sigma_{\varepsilon}^{2}=0.1$. Here, we assume that the maximum delay $n_{y}$ and $n_{u}$ are known for simplicity.

Table 1: GA configurations

\begin{tabular}{lcc}
\hline & multiobjective GA & UNDX \\
\hline crossover rate & 0.7 & - \\
mutation rate & 0.1 & - \\
population size & 50 & 30 \\
\hline
\end{tabular}

The MSE values are shown in the Table. 2 and the trajectories of the observed output and predicted output by using provided ensemble model are indicated in Fig.7, 8 and 9 .

Table 2: Numerical simulation results (1)

\begin{tabular}{ccc}
\hline \multirow{2}{*}{ RBF width $\left(\sigma^{2}\right)$} & \multicolumn{2}{c}{ Number of generations } \\
\cline { 2 - 3 } & 5 & 50 \\
\hline 1.0 & 0.201851 & 0.202434 \\
\hline 2.0 & 0.198471 & 0.201319 \\
\hline 5.0 & 0.210963 & 0.214082 \\
\hline
\end{tabular}

\subsection{Simulation 2}

Then, we assume that the true system is described by

$$
\begin{aligned}
y_{t}= & -0.5 y_{t-2}+0.7 u_{t-1} y_{t-1}+0.6 u_{t-2}^{2} \\
& +0.2 y_{t-1}^{3}-0.7 u_{t-2}^{2} y_{t-2} .
\end{aligned}
$$

We carried out simulation runs with same condition as Simulation 1 . but the maximum delay $n_{y}$ and $n_{u}$ are assumed 2 and 3 , respectively.

The MSE values are shown in the Table. 2 and the trajectories of the observed output and predicted output by using provided ensemble model are indicated in Fig.10, 11 and 12 .

Table 3: Numerical simulation results (2)

\begin{tabular}{cccc}
\hline \multirow{2}{*}{ RBF width $\left(\sigma^{2}\right)$} & delay & \multicolumn{2}{c}{ Number of generations } \\
\cline { 2 - 4 } & 2 & 5 & 50 \\
\hline \multirow{2}{*}{1.0} & 3 & 0.25170057 & 0.189671 \\
\hline \multirow{2}{*}{2.0} & 2 & 0.169839 & 0.250289 \\
\hline & 3 & 0.213453 & 0.212877 \\
\hline 5.0 & 2 & 0.197117 & 0.192981 \\
\hline & 3 & 0.223191 & 0.218099 \\
\hline
\end{tabular}

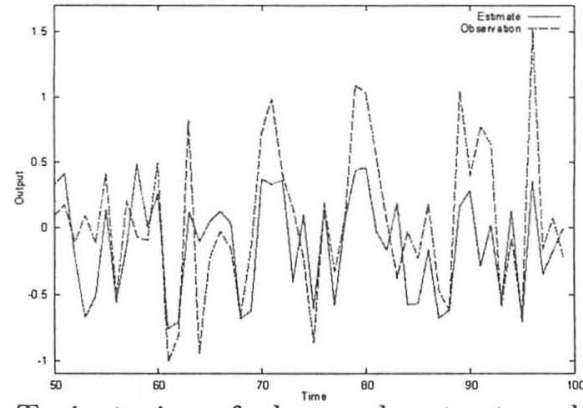

Fig. 7: Trajectories of observed output and one step prediction by the provided model $\left(\sigma^{2}=1.0\right)$

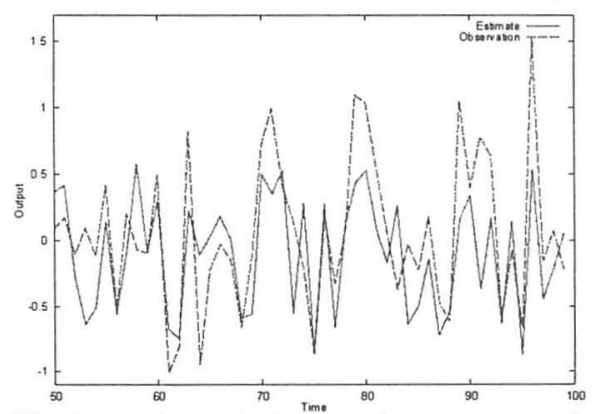

Fig. 8: Trajectories of observed output and one step prediction by the provided model $\sigma^{2}=2.0$

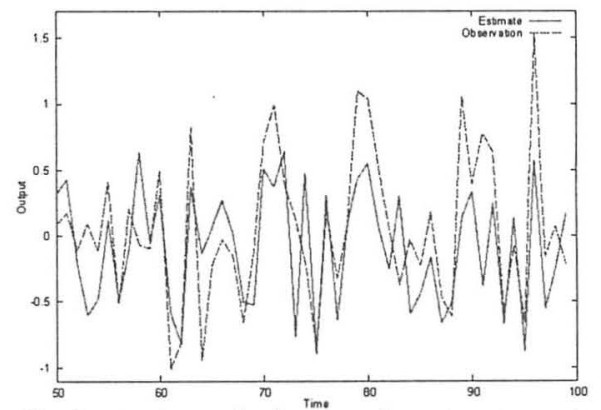

Fig. 9: Trajectories of observed output and one step prediction by the provided model $\sigma^{2}=5.0$

\section{Conclusions}

In this study, we have proposed a method of obtaining a Pareto optimal RBF network set based on multiobjective evolutionary algorithms and constructing an ensemble network by such Pareto optimal networks. Then, an application of the ensemble network to nonlinear system identification is also considered. $\mathrm{Nu}-$ merical simulation results indicate that the ensemble network has an ability to identify nonlinear systems without specific prior knowledge or information about the objective systems. Reduction of the computational costs, improvement of the ensemble technique and comparison to the conventional approaches are the future works and they are under investigation.

\section{References}

[1] L. Ljung : System Identification: Theory for the User, Prentice-Hall, NJ, 1999. 


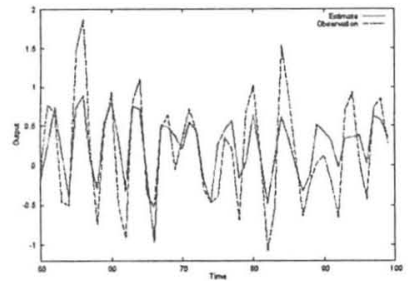

(a) $n_{y}=n_{u}=2$

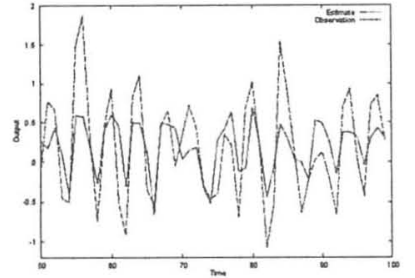

(b) $n_{y}=n_{u}=3$
Fig. 10: trajectories of observed output and one step prediction by the provided model $\sigma^{2}=1.0$

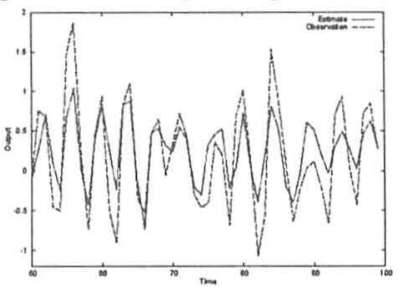

(a) $n_{y}=n_{u}=2$

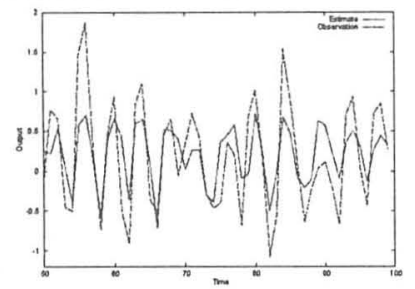

(b) $n_{y}=n_{u}=3$
Fig. 11: trajectories of observed output and one step prediction by the provided model $\sigma^{2}=2.0$ (a) $n_{y}=n_{u}=2$

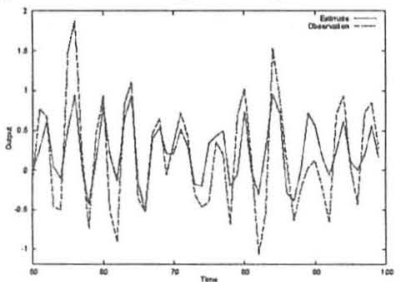

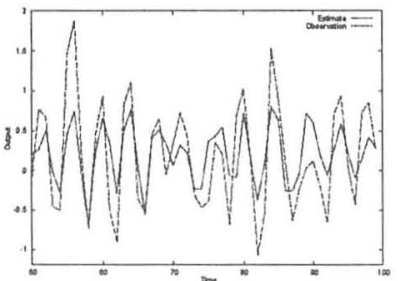

(b) $n_{y}=n_{u}=3$
Fig. 12: trajectories of observed output and one step prediction by the provided model $\sigma^{2}=5.0$

[2] O. Nelles : Nonlinear System Identification, Springer, Berlin, 2000.

[3] T. A. Johansen : Multi-objective identification of FIR models, Proceedings of IFAC Symposium on System Identification SYSID2000, Vol.3, pp.917922, 2000.

[4] T. A. Johansen and R. Babuška: Multi-Objective Identification of Takagi-Sugeno Fuzzy Models, IEEE Transactions on Fuzzy Systems, Vol.11, No.6, pp.847-860, 2003.

[5] C. M. Fonseca and P. J. Fleming, P. J : Genetic algorithms for multiobjective optimization : Formulation, discussion and generalization, Proceedings of the Fifth International Conference on Genetic Algorithms, pp.416-423, 1993.

[6] K. Deb : Multi-objective Optimization using Evolutionary Algorithms, John Wiley \& Sons, New York, 2001.

[7] R.-V. Katya C. M. Fonseca and P. J. Flemming : Multiobjective Genetic Programming : A NonLinear System Identification Application, Genetic Programming 97 Conference, pp.207-212, 1997.
[8] T. Hatanaka, K. Uosaki and Y. Hossaka : Application of Evolutionary Multi-Objective Optimization to Nonlinear System Identification, Proceedings of the SICE Kansai Branch Symposium 2002, pp.5456, 2002. (in Japanese)

[9] S. Chen and S. A. Billings : Neural networks for nonlinear dynamic system modelling and identification, International Journal of Control, Vol.56, No.2, pp. 319-346, 1992.

[10] J. Sjoberg, Q. Zhang et.al. : Nonlinear blackbox modeling in system identification : a unified overview, Automatica, Vol.31, No.12, pp.16911724,1995 .

[11] T. Hatanaka, K. Uosaki and T. Kuroda : Structure Selection of RBF Neural Network Using Information Criteria, Proceedings of Fifth International Conference on Knowledge-Based Intelligent Information Engineering Systems and Allied Technologies, pp.166-170, 2001.

[12] Y. Jin, T. Okabe and B. Sendhoff : Neural network regularization and ensembling using multiobjective evolutionary algorithms, Proceedings of the 2004 IEEE Congress on Evolutionary Computation, pp.1-8, 2004

[13] M.M. Islam, X. Yao and K. Murase : A constructive algorithm for training cooperative neural network ensembles, IEEE Transactions on Neural Networks, Vol.14, No.4, pp.820-834, 2003.

[14] H. A. Abbass : Pareto Neuro-Ensemble, Proceedings of the 16th Australian Joint Conference on Artificial Intelligence, pp.554-566, 2003.

[15] H. A. Abbass : Pareto Neuro-Evolution : Constructing Ensemble of Neural Networks Using Multi-objective Optimization, Proceedings of the 2003 IEEE Congress on Evolutionary Computation, Vol.3, pp.2074-2080, 2004.

[16] J. E. Fieldsend and S. Singh : Pareto evolutionary neural networks, IEEE Transactions on Neural Networks, Vol.16, No.2, pp.338-354, 2005.

[17] J. Holland : Adaption in Natural and Artificial Systems, The University of Michigan Press, MI, 1975.

[18] I. Ono and S. Kobayashi : A Real-coded Genetic Algorithm for Function Optimization Using Unimodal Normal Distribution Crossover, Proceedings of 7th International Conference.on Genetic Algorithms, pp.246-253, 1997.

[19] H. Sato, I. Ono and S. Kobayashi : A New Generation Alternation Model of Genetic Algorithms and Its Assessment, Journal of Japanese Society for Artificial Intelligence, Vol.12, No.5, pp.734744, 1997. 